\title{
N100, N200, and P300 Auditory Event-Related Potentials Depending on Handedness and Response Tasks such as Button Pressing and Mental Counting
}

\author{
Jinsook Kim ${ }^{1,2}$, Kieun Lee ${ }^{1}$, Eunsung Lee ${ }^{1}$ \\ 'Department of Speech Pathology and Audiology, Graduate School, Hallym University, Chuncheon, Korea \\ ${ }^{2}$ Division of Speech Pathology and Audiology, Research Institute of Audiology and Speech Pathology, College of Natural Sciences, Hallym University, \\ Chuncheon, Korea
}

Received: August 22, 2020

Revised: October 3, 2020

Accepted: October 10, 2020

\section{Correspondence:}

Jinsook Kim, PhD

Division of Speech Pathology and

Audiology, Research Institute

of Audiology and Speech Pathology,

College of Natural Sciences,

Hallym University,

1 Hallymdaehak-gil

Chuncheon 24252, Korea

Tel: +82-33-248-2213

Fax: +82-33-256-3240

E-mail: jskim@hallym.ac.kr

\begin{abstract}
Purpose: This study was to determine the effects of response tasks, such as button pressing and mental counting, and handedness on N100, N200, and P300 auditory event-related potential (AERP). Methods: A total of 50 normal-hearing young adults with the average age of $21.6( \pm 1.5)$ years participated in this study. Among them, 15 men and 15 women were right-handed and 10 men and 10 women were left-handed. An oddball paradigm was used to deliver 30 stimuli of $2 \mathrm{kHz}$ target tone bursts and 120 stimuli of $1 \mathrm{kHz}$ nontarget tone bursts. The stimuli were presented at 70 $\mathrm{dB}$ sound pressure level with the rate of $1 / \mathrm{s}$. Results: The button pressing task elicited significantly smaller N100 and larger P300 amplitudes than the mental counting task. N200 latency was significantly lower and P300 amplitude was higher in left-handed participants than those who are right-handed. Appearance percentages of right-/left-handed participants for N100, N200, and P300 were 80/95\%, 85/85\%, and 75/75\% for the button pressing task and 80/90\%, 80/80\%, and $70 / 70 \%$ for mental counting task, respectively. Conclusion: The significant difference in appearance percentage between response tasks supported that P300 was a strong endogenous potential. $\mathrm{N} 100$ and N200 were thought to have both endogenous and exogenous characteristics. A more sensitive approach in selecting the task of response for the target stimuli and careful consideration for the handedness is necessary for AERP recordings.
\end{abstract}

Key Words: Event-related potentials, Handedness, Response tasks.

\section{INTRODUCTION}

The auditory event-related potential (AERP) is a time-locked tool to measure the electrical activity of the cerebral surface representing a distinct phase of cortical processing. The first N100 component has been conceptualized as a pre-attentive response related to perception. The N100 response was modulated by the physical characteristics of stimuli such as type, intensity, and duration, and also depended on subject factors such as the internal state, neural activity, and cognition. As it was the first stimulus in a series, the N100 amplitude was relatively high but lowered for subsequent repetitions of stimuli (Brown et al., 2002; Clearwater et al., 2008). N200 has been known to be a

(c) This is an Open Access article distributed under the terms of the Creative Commons Attribution Non-Commercial License (https://creativecommons.org/licenses/by-nc/4.0) which permits unrestricted non-commercial use, distribution, and reproduction in any medium, provided the original work is properly cited. comprehensive response of cortical, thalamic, and brainstem regions, although the source of N200 activation relied on some extent to the limbic and complex generating system in the region of the thalamus (Ponton et al., 2002). N200 was also thought to be sensitive to cognitive aspects such as task demands and attention (Näätänen \& Picton, 1986). However, the latency of N200 exhibited conflicting findings depending on the age of participants (Amenedo \& Díaz, 1998). As observed only during conscious stimulus attention, P300 was often found as a part of complex components of N200, which were categorized as endogenous potentials because even mere mental counting tasks for target stimuli could elicit them well (Kaga et al., 2014). P300 reflected the brain activity required to update working memory when changes occurred in the stimulus and variation of the amplitude indicated the degree of inclusion of the central nervous system activity involved in processing information (Verleger et al., 2014). 
Several factors affecting P300 sensitivity were observed in the literature regarding age, handedness, personality traits, and cognitive abilities. Increased P300 latency has been reportedly associated with increasing age, whereas decreased amplitude was associated with extraverted and low cognitive abilities (Anderer et al., 1996; Ivry \& Lebby, 1993).

The handedness influenced the fundamental cognitive mechanisms and consequently altered AERP components. Specifically, larger callosal pathways in left-handed participants were reported, and such hemispheric difference resulted in a larger P300 amplitude (Hoffman \& Polich, 1999). However, N100 and N200 did not show any handedness differences. Regarding response tasks, such as button pressing and mental counting for target stimuli, discrepant findings among investigators were observed. The larger P300 amplitude in button pressing task was found to reflect more coherent synaptic activity in response execution (Brázdil et al., 2003) ; however, decreased in both P300 amplitude and latency were observed by other researchers. Decreased P300 response was explained by the smaller amount of attentional resources, melding of P300 responses, and coincidental movement-related potentials in summative scalp recordings for the button pressing task (Barrett et al., 1987; Salisbury et al., 2001). In addition, P300 responses were reportedly affected by sex due to existing neuroanatomical and functional differences in the brain between women and men. The larger P300 amplitude was observed in the wider range of structural and physiological aspects of the female brain, with the difference in thickness, fashion of brain wiring, and pattern of the hemisphere connectome (Melynyte et al., 2018). Besides sex influence, the P300 amplitude was significantly affected by the handedness with larger corpus callosal areas of left-handed participants proven by a meta-analysis (Polich \& Hoffman, 1998).

Apparently, AERP responses were influenced by the neural synaptic activity and hemisphere related callosal size. Effects of response tasks, handedness, and sex were of interest when the target and nontarget tones were presented in an oddball paradigm to elucidate the electrophysiological mechanism of the central auditory processing. This study aimed to determine whether and how AERP components were affected by response tasks, handedness, and sex. The hypothesis was established that the differences in N100, N200, and P300 responses according to the task of response would be evident. And as the size of the corpus callosum was larger in the left-handed and the female, they would show the larger amplitude for N100, N200, and P300 responses. By comparing the results, the endogenous and exogenous characteristics of N100, N200, and P300 could be further understood. Also, the impact of brain-related characteristics for those could be investigated more deeply.

\section{MATERIALS AND METHODS}

\section{Participants}

A total of 50 normal-hearing young adults with an average age of $21.6( \pm 1.5)$ years were recruited among university students. All participants read and signed the consent document. An approval of research protocols was obtained before the research process began (\#HIRB-2018-044). Right-handed participants consisted of 15 men and 15 women with an average age of $21.9( \pm 1.2)$ years, and left-handed participants consisted of 10 men and 10 women with an average age of $21.3( \pm 1.5)$ years. The left-handed was confirmed when the participant used left hands in general life mostly including writing and eating.

\section{Sound stimuli}

Bio-logic Navigator Pro (Natus, Mundelin, IL, USA) was used to generate acoustic stimuli and record AERP. The sound stimuli were an envelope of 1-100-1 ms of a tone burst. An oddball paradigm was used to deliver target and nontarget tone bursts. Target tones were 30 rare stimuli of $2 \mathrm{kHz}$ tone bursts, and nontarget tones were 120 frequent stimuli of $1 \mathrm{kHz}$ tone bursts. The ratio of target to nontarget stimuli was 4:1. A total of 150 stimuli were presented to each participant in random order. All stimuli were delivered at a rate of $1 / \mathrm{s}$, and a bandpass filter was set at $0.5-100 \mathrm{~Hz}$. Participants were stimulated binaurally using supra-aural headphones TDH-39 (Telephonics, Huntington, WV, USA) in the right and left ears simultaneously at $70 \mathrm{~dB}$ sound pressure level.

\section{Recording parameters}

To analyze AERPs, the bipolar channel was used, channel A for measuring AERPs and channel B for rejecting eye movement and blinking. For channel A, the reference electrode was placed at $\mathrm{A} 1$ and $\mathrm{A} 2$, the active electrode at $\mathrm{Cz}$, and the common ground electrode at the Fpz. For channel B, the reference electrode was placed at the left infraorbital, and the active electrode was placed at the left supraorbital. The impedance was maintained below $5 \mathrm{k} \Omega$ for all electrode positions. Artifacts were automatically rejected at $\pm 60 \mu$.

\section{Procedures}

Button pressing and mental counting tasks were used to obtain responses for target tones. The correction rate was almost 
$100 \%$ as the participants were normal hearing young students. Whether they were the left-handed or right-handed, button pressing tasks were performed with both right thumb and left thumbs separately. In the mental counting, the number of targets was confirmed by counting the number of targets instead of pressing the button whenever the target tone was heard. As the target stimuli were heard to the participants in a relaxed position while lying on the bed with closed eyes but remaining awake, the right thumb button pressing, left thumb button pressing, and mental counting were performed randomly.

\section{Statistical analysis}

Amplitudes and latencies of AERP depending on the task of response, handedness, and sex were analyzed statistically with the Statistical Package for the Social Sciences (ver.25.0, IBM Corp., Armonk, NY, USA) software. Two-way analysis of variance was used for statistical analysis and a significant level was defined at $p$-value of 0.05 .

\section{RESULTS}

Grand averages of AERP of target and nontarget tones in the button pressing and mental counting tasks depending on the handedness are shown in Figure 1. The statistical significance of the main effect was revealed in the handedness and the task of response (Table 1). Left-handed participants showed shorter N200 latency and larger P300 amplitude. In addition, the button pressing task showed smaller N100 and larger P300 amplitudes (Figure 2). When compared to the use of the right and left thumbs for the button pressing task, the result did not show any statistical significance.

The statistical significance of the interaction effects found in handedness $\times$ task of response, handedness $\times$ sex, handedness $\times$ use of thumbs, and handedness $\times$ use of thumbs $\times$ sex show

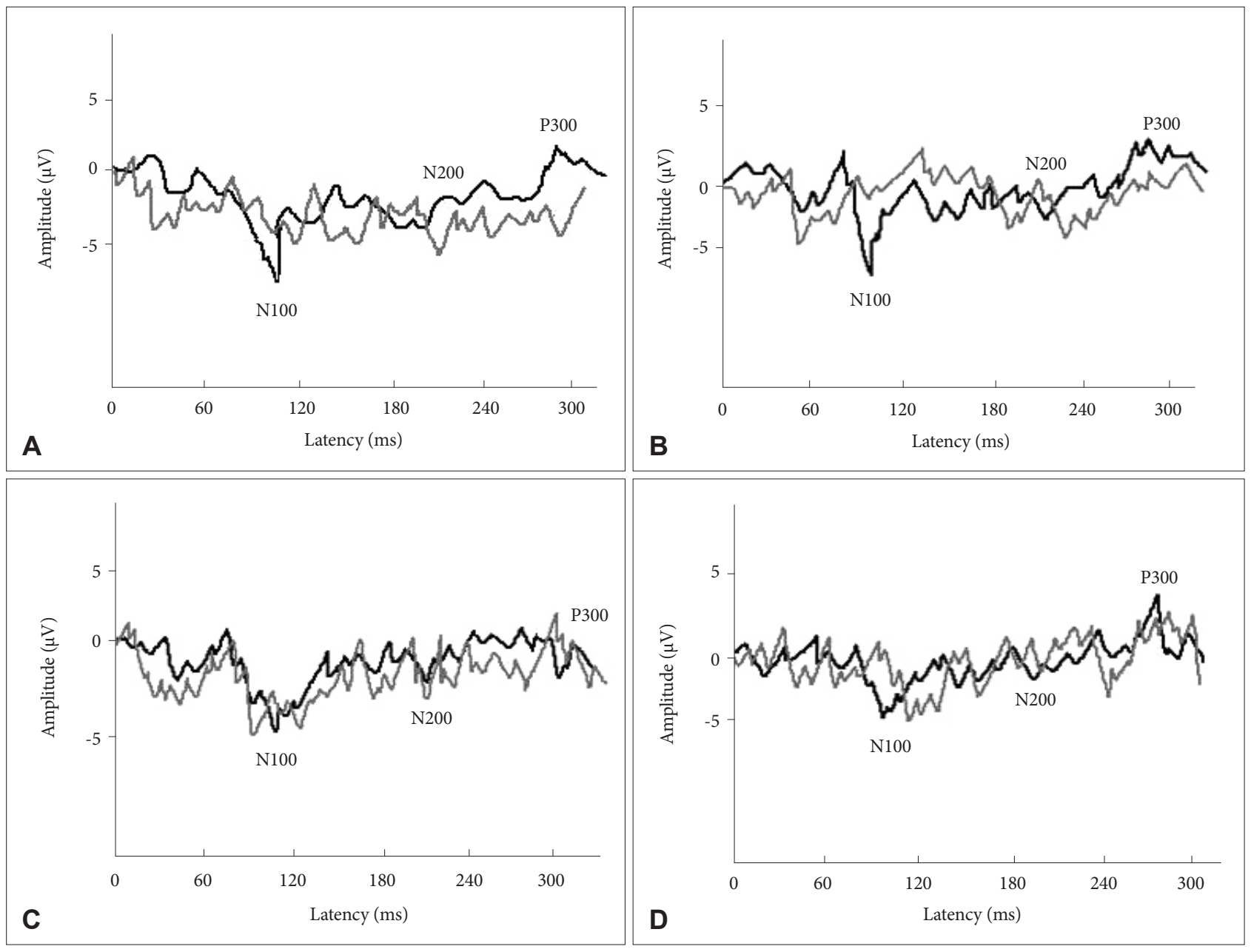

Figure 1. Grand averages of auditory event-related potential of target and nontarget tones according to handedness and response method. black bold lines depict responses of target tones and grey lines depict responses of nontarget tones. Button pressing for right handed (A), button pressing for left handed (B), mental counting for right handed (C), mental counting for left handed (D). 
Table 1. Summary of statistical analysis of variance for main and interaction effects

\begin{tabular}{|c|c|c|c|c|c|c|}
\hline & \multicolumn{3}{|c|}{ Latency (ms) } & \multicolumn{3}{|c|}{ Amplitude $(\mu \mathrm{V})$} \\
\hline & N100 & N200 & P300 & N100 & $\mathrm{N} 200$ & P300 \\
\hline \multicolumn{7}{|l|}{ Main effect } \\
\hline Handedness (R, L) & 0.680 & $0.037^{*}$ & 0.606 & 0.070 & 0.768 & $0.025^{*}$ \\
\hline Task of response (BP, MC) & 0.140 & 0.842 & 0.982 & $0.002^{*}$ & 0.199 & $0.011^{*}$ \\
\hline Use of thumbs (BPR, BPL) & 0.244 & 0.487 & 0.740 & 0.996 & 0.898 & 0.559 \\
\hline $\operatorname{Sex}(M, F)$ & 0.719 & 0.983 & 0.863 & 0.139 & 0.456 & 0.907 \\
\hline \multicolumn{7}{|l|}{ Interaction effect } \\
\hline Handedness $\times$ task of response & 0.392 & 0.323 & 0.508 & 0.727 & $0.040^{*}$ & 0.170 \\
\hline Use of thumbs $\times$ sex & 0.423 & 0.453 & 0.880 & 0.384 & 0.471 & 0.678 \\
\hline Handedness $\times$ sex & 0.702 & 0.071 & 0.751 & 0.081 & 0.100 & $0.003^{*}$ \\
\hline Task of response $\times$ sex & 0.464 & 0.350 & 0.461 & 0.961 & 0.784 & 0.119 \\
\hline Handedness $\times$ use of thumbs & $0.003^{*}$ & 0.832 & 0.144 & 0.415 & 0.146 & 0.082 \\
\hline Handedness $\times$ use of thumbs $\times$ sex & $0.010^{*}$ & 0.465 & 0.227 & $0.011^{*}$ & 0.837 & $0.000^{*}$ \\
\hline
\end{tabular}

${ }^{*} p<0.05$. BP: button pressing, MC: mental counting, BPR: button pressing right, BPL: button pressing left

Figure 2. Amplitudes of auditory eventrelated potential components according to $\mathrm{BP}$ and $\mathrm{MC}$ tasks between $\mathrm{LH}$ and $\mathrm{RH}$ participants. BP: button pressing, MC: mental counting, $\mathrm{LH}$ : left-handed, $\mathrm{RH}$ : right-handed.

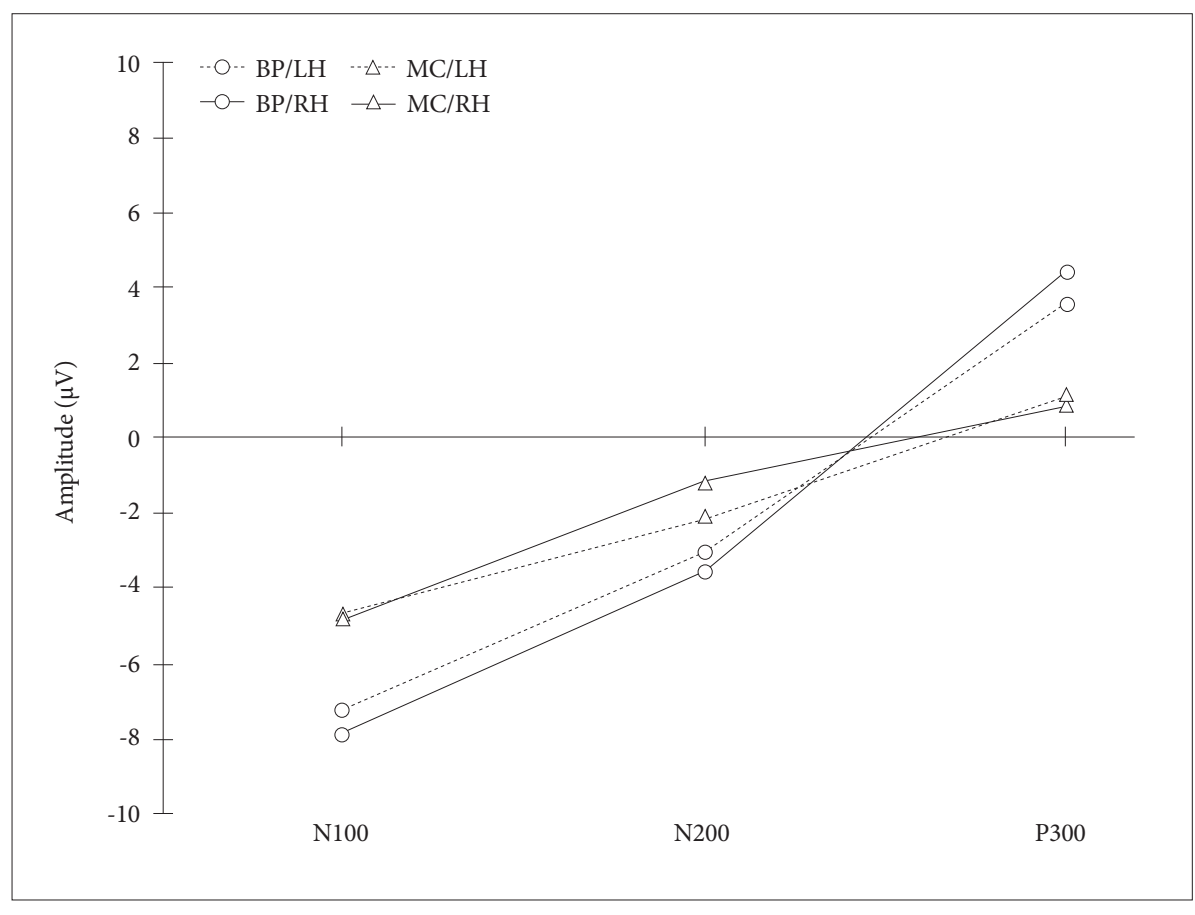

ance percentage in the mental counting task for P300 of both right- and left-handed participants (Figure 3).

\section{DISCUSSIONS}

Our study revealed significant differences in the handedness and task responses. Smaller N100 and larger P300 amplitudes for the button pressing task and shorter N200 latency and larger P300 amplitude of left-handed participants were found. The N100 amplitude was relatively large as it was the first stimulus in a series, but it became smaller due to subsequent stimulus repetitions (Clearwater et al., 2008; Michalski, 2000). However, this sequen- 
Table 2. Average of latency (ms) for auditory event-related potential components according to BPR, BPL and MC tasks

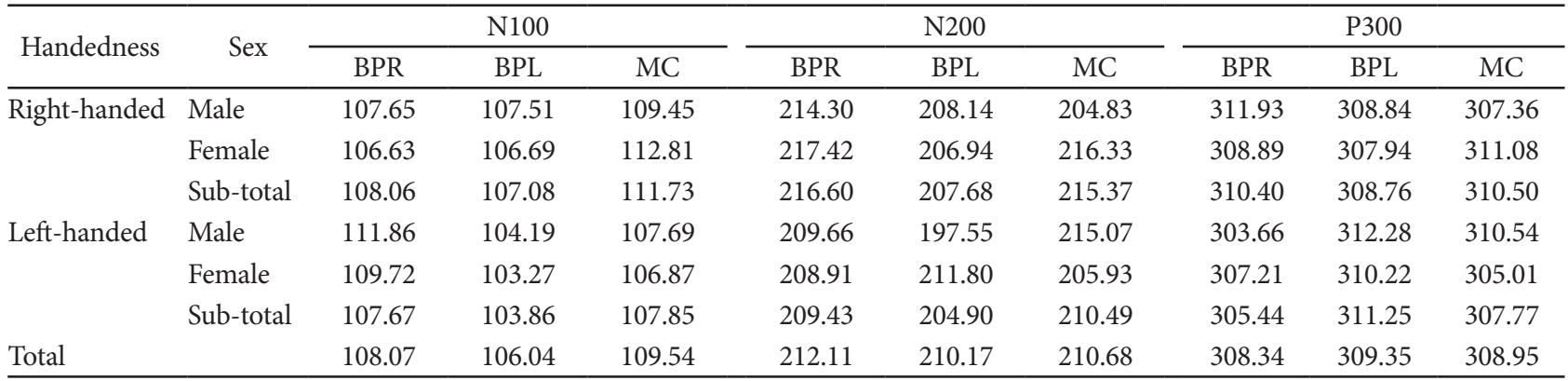

BPR: button pressing right, BPL: button pressing left, MC: mental counting

Table 3. Average of amplitude $(\mu \mathrm{V})$ for auditory event-related potential components according to BPR, BPL and MC tasks

\begin{tabular}{|c|c|c|c|c|c|c|c|c|c|c|}
\hline \multirow{2}{*}{ Handedness } & \multirow{2}{*}{ Sex } & \multicolumn{3}{|c|}{ N100 } & \multicolumn{3}{|c|}{ N200 } & \multicolumn{3}{|c|}{ P300 } \\
\hline & & $\mathrm{BPR}$ & $\mathrm{BPL}$ & $\mathrm{MC}$ & $\mathrm{BPR}$ & $\mathrm{BPL}$ & $\mathrm{MC}$ & BPR & $\mathrm{BPL}$ & $\mathrm{MC}$ \\
\hline \multirow[t]{3}{*}{ Right-handed } & Male & -7.20 & -8.89 & -5.75 & -2.80 & -2.48 & -0.86 & 1.01 & 1.05 & 1.30 \\
\hline & Female & -6.84 & -5.75 & -4.12 & -3.79 & -3.39 & -2.16 & 1.08 & 1.24 & 1.37 \\
\hline & Sub-total & -7.93 & -7.89 & -4.77 & -3.54 & -3.16 & -1.14 & 1.12 & 2.52 & 0.92 \\
\hline \multirow[t]{3}{*}{ Left-handed } & Male & -5.82 & -7.14 & -4.69 & -2.99 & -3.56 & -3.00 & 0.70 & 1.64 & 1.30 \\
\hline & Female & -5.74 & -6.23 & -4.81 & -1.18 & -3.25 & -2.84 & 4.67 & 5.56 & 1.37 \\
\hline & Sub-total & -5.79 & -6.80 & -4.84 & -2.08 & -3.40 & -2.92 & 2.68 & 3.60 & 1.34 \\
\hline Total & & -7.25 & -7.39 & -4.64 & -2.93 & -3.05 & -2.08 & 3.59 & 3.05 & 1.16 \\
\hline
\end{tabular}

BPR: button pressing right, BPL: button pressing left, MC: mental counting

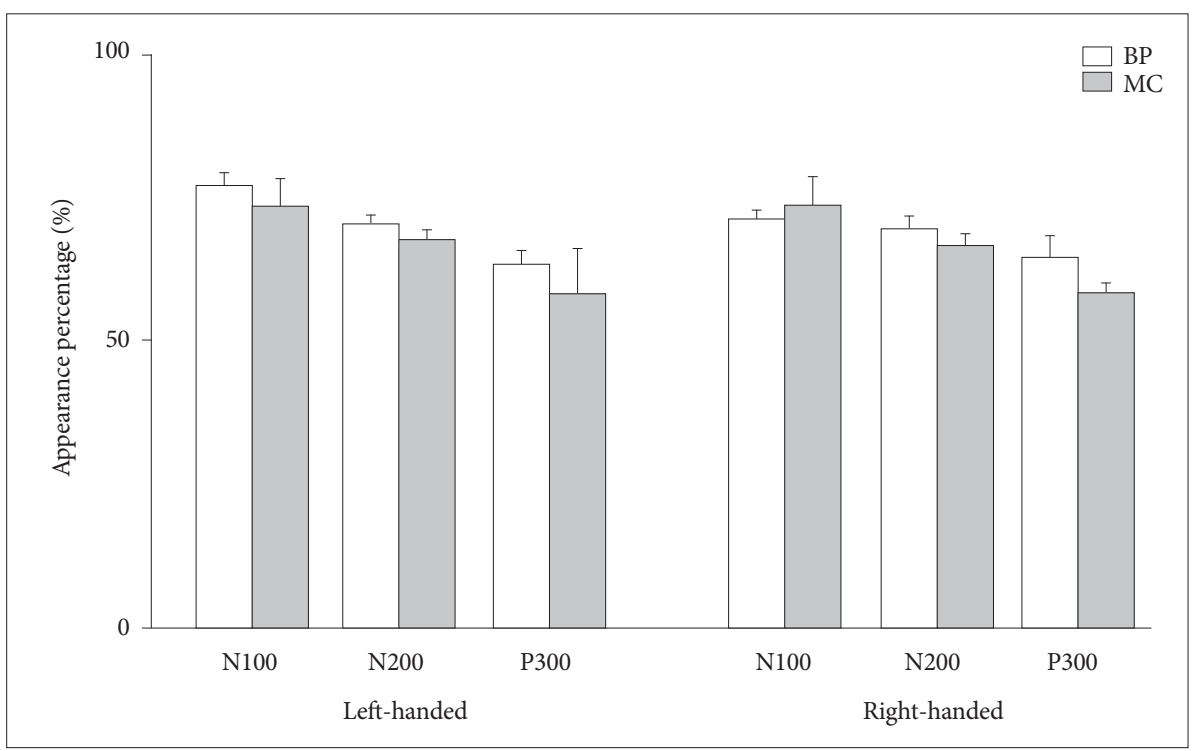

Figure 3. Comparison of appearance percentages of N100, N200, and P300 between $\mathrm{BP}$ and $\mathrm{MC}$ tasks for target tone. BP: button pressing, MC: mental counting. tial effect diminished and was less clear with the mental counting task (Starr et al., 1997) and a larger N100 amplitude was found in the mental counting task in this study. The larger P300 amplitude for the button pressing task coincided with previous studies, reflecting the higher degree of synchronization of activated neurons and more coherent synaptic activity in response execution (Brázdil et al., 2003; Kaga et al., 2014). On the contrary, melding of the later movement of coincident P300-like potentials of true P300 activity has been reported to elicit smaller P300 amplitude with the button pressing task (Brázdil et al., 2003; Salisbury et al., 2001). Moreover, considering the age effect to the cognition, wider age range might have played a role for the smaller P300 amplitude in a previous study with the age range of 18-53 years (Salisbury et al., 2001) comparing to the age range of 2028 in this study. In addition, the amplitude of P300 becomes smaller as the task complexity enhancement (Polich, 1987). Dur- 
ing mental counting, participants had to remember target tones, which was more difficult than the button pressing task. This finding suggested that the P300 amplitude obtained from auditory paradigms reflected processing difficulty independent of the stimulus target probability. Also, the mental counting task could be accompanied by subtle activation of muscles of the face, tongue, and larynx, which might have affected the smaller P300 amplitude (Starr et al., 1995). Such small and unapparent movements could have led to the generation of the smaller amplitude during mental counting.

Regardless of response tasks, the lowest appearance percentage was observed in P300 supporting that P300 has psychological characteristics. Early components of AERP of this study exhibited higher response percentages than P300, which might have been affected by the degree of understanding and age of participants. The lower appearance percentage of P300 for the mental counting task of right-/left-handed participants was observed when compared to the button pressing task. This was agreed with the previous study showing $30 \%$ of appearance percentage for the mental counting task and $90 \%$ of appearance percentage for the button pressing (Kaga et al., 2014). The reason for the lower percentage of mental counting task was thought to be related to negative factors such as adaptation, habituation, and mental fatigue as mental counting task was easily affected by negative factors. The slightly larger or similar appearance percentages for the task of button pressing of right-/left-handed participants were observed in N100 and N200 when compared to mental counting task in this study. As the previous study noted, no habituation effect in these early components was found (Ravden \& Polich, 1998). In addition, as the appearance percentage of early components were not affected by the response tasks, it was difficult to determine whether they had the endogenous and/or exogenous characteristics.

It was reported that the $\mathrm{N} 200$ latency was shorter at anterior locations for left-handed for the target stimulus in the previous research (Alexander \& Polich, 1997) which was agreed with the results of this study. However, the N200 amplitude did not produce any reliable handedness effects. The larger P300 amplitude in left-handed participants was also reported with the visual stimuli (Ravden \& Polich, 1998). Handedness, which appeared to stem from genetic neuroanatomical differences had also been found to be associated with cerebral size asymmetries for leftand right-handed participants. Through the magnetic resonance imaging data, it was identified that the left-handed participants had larger callosal areas (Habib et al., 1991). Individuals with inherently larger callosal size should demonstrate larger P300 am- plitudes (Alexander \& Polich, 1997). Therefore, left-handed participants in this study whose callosal size was thought to be larger showed the larger P300 amplitude with neuroanatomical and functional dissimilarities. Similarly, the larger size of the corpus callosum in female was also reported to contribute to the larger amplitude and the shorter latency of P300 in the literature (Melynyte et al., 2018; Schiff et al., 2008). In addition, there was lateralization of nerve conduction velocity between left- and right-handed participants (Gupta et al., 2008). The asymmetry of sensory conduction velocity depending on the handedness due to genetic reasons was reported in the literature (Bromberg \& Jaros, 1998; Gupta et al., 2008). This factor was recommended to be taken into account for making any neurological diagnosis in the left-handed as this might have contributed to the functional differences by the previous researcher. Also, the left-handed reacted faster for the auditory stimulus when the reaction time was evaluated (Sathiamoorthy et al., 1994). Therefore, it should be further studied to identify the relationship between the AERP response of handedness depending on nerve conduction velocity as the previous researcher recommended. This study has a few limitations. The responses of various age groups including 30$60 \mathrm{~s}$, and a large number of participants would provide more comprehensive data. But the age group was only at 20 s and the number of participants were only 50 in this study. Also, it was unfortunate to include only the students who knew the test protocol well and the handedness questionnaire such as 'Edingurgh Handedness Questionnaire' was not applied for the selection of the left- handed participants in this study. However, the left-handed participants answered that they used left hands for the general activity in daily life including writing and eating as the questionnaire included standard activities of the left-handed. More various groups and careful selection of participants should be considered in the future study.

P300 might be an exogenous potential if it depended on physical variables. However, the test parameters which were considered as a physical variable were constant in this study, and there was a change of P300 in handedness and response task, which seemed to be an endogenous potential because it showed that it represented psychological characteristics. But it was difficult to determine whether the early components had the endogenous and/or exogenous characteristics through this study. As N100, $\mathrm{N} 200$, and P300 responses might be differed by handedness and tasks of response, a more sensitive approach for selecting the task of response for the target stimuli and careful consideration for the handedness of participants are recommended. 


\section{Ethical Statement}

All participants signed an informed consent form before conducting the experiments. The protocol of this study has been approved by the Institutional Review Board of Hallym University (HIRB-2018-044).

\section{Acknowledgments}

The authors thank the students of Hallym University for participating in this research.

\section{Declaration of Conflicting Interests \\ There are no conflict of interests.}

\section{Funding}

This work was supported by Hallym University Research Funds (HRF201808-008)

\section{Author Contributions}

J.K. and K.L. designed and experiment together. K.L. collected data and K.L and E.L. analyzed the data. J.K. and K.L. wrote the draft for the manuscript, and then J.K. revised the manuscript as the corresponding author. All authors discussed at each stage for the manuscript while providing critical comments.

\section{ORCID iD}

Jinsook Kim

$$
\text { https://orcid.org/0000-0003-3440-2393 }
$$

\section{REFERENCES}

Alexander, J. E. \& Polich, J. (1997). Handedness and P300 from auditory stimuli. Brain and Cognition, 35(2), 259-270.

Amenedo, E. \& Díaz, F. (1998). Aging-related changes in processing of nontarget and target stimuli during an auditory oddball task. Biological Psychology, 48(3), 235-267.

Anderer, P., Semlitsch, H. V., \& Saletu, B. (1996). Multichannel auditory event-related brain potentials: Effects of normal aging on the scalp distribution of N1, P2, N2 and P300 latencies and amplitudes. Electroencephalography and Clinical Neurophysiology, 99(5), 458-472.

Barrett, G., Neshige, R., \& Shibasaki, H. (1987). Human auditory and somatosensory event-related potentials: Effects of response condition and age. Electroencephalography and Clinical Neurophysiology, 66(4), 409419.

Brázdil, M., Roman, R., Daniel, P., \& Rektor, I. (2003). Intracerebral somatosensory event-related potentials: Effect of response type (button pressing versus mental counting) on P3-like potentials within the human brain. Clinical Neurophysiology, 114(8), 1489-1496.

Bromberg, M. B. \& Jaros, L. (1998). Symmetry of normal motor and sensory nerve conduction measurements. Muscle and Nerve, 21(4), 498-503.

Brown, K. J., Gonsalvez, C. J., Harris, A. W. F., Williams, L. M., \& Gordon, E. (2002). Target and non-target ERP disturbances in first episode vs. chronic schizophrenia. Clinical Neurophysiology, 113(11), 1754-1763.

Clearwater, J. M., Kerr, C. C., Rennie, C. J., \& Robinson, P. A. (2008). Neural mechanisms of ERP change: Combining insights from electrophysiology and mathematical modeling. Journal of Integrative Neuroscience, 7(4), 529-550

Gupta, N., Sanyal, S., \& Babbar, R. (2008). Sensory nerve conduction velocity is greater in left handed persons. Indian Journal of Physiology and Pharmacology, 52(2), 189-192.
Habib, M., Gayraud, D., Oliva, A., Regis, J., Salamon, G., \& Khalil, R. (1991). Effects of handedness and sex on the morphology of the corpus callosum: A study with brain magnetic resonance imaging. Brain and Cognition, 16(1), 41-61.

Hoffman, L. D. \& Polich, J. (1999). P300, handedness, and corpus callosal size: Gender, modality, and task. International Journal of Psychophysiology, 31(2), 163-174.

Ivry, R. B. \& Lebby, P. C. (1993). Hemispheric differences in auditory perception are similar to those found in visual perception. Psychological Science, 4(1), 41-45

Kaga, K., Fukami, T., Masubuchi, N., \& Ishikawa, B. (2014). Effects of button pressing and mental counting on N100, N200, and P300 of auditory-event-related potential recording. Journal of International Advanced Otology, 10(1), 14-18.

Melynyte, S., Wang, G. Y., \& Griskova-Bulanova, I. (2018). Gender effects on auditory P300: A systematic review. International Journal of Psychophysiology, 133, 55-65.

Michalski, A. (2000). Expectation of an important event affects responses to irrelevant stimuli of different modalities. Acta neurobiologiae Experimentalis, 60(4), 467-478.

Näätänen, R. \& Picton, T. W. (1986). N2 and automatic versus controlled processes. Electroencephalography and Clinical Neurophysiology Supplement, 38, 169-186.

Polich, J. \& Hoffman, L. D. (1998). P300 and handedness: On the possible contribution of corpus callosal size to ERPs. Psychophysiology, 35(5), 497-507.

Polich, J. (1987). Task difficulty, probability, and inter-stimulus interval as determinants of P300 from auditory stimuli. Electroencephalography and Clinical Neurophysiology, 68(4), 311-320.

Ponton, C., Eggermont, J. J., Khosla, D., Kwong, B., \& Don, M. (2002). Maturation of human central auditory system activity: Separating auditory evoked potentials by dipole source modeling. Clinical Neurophysiology, 113(3), 407-420.

Ravden, D. \& Polich, J. (1998). Habituation of P300 from visual stimuli. International Journal of Psychophysiology, 30(3), 359-365.

Salisbury, D. F., Rutherford, B., Shenton, M. E., \& McCarley, R. W. (2001). Button-pressing affects P300 amplitude and scalp topography. Clinical Neurophysiology, 112(9), 1676-1684.

Sathiamoorthy, A., Sathiamoorthy, S. S., Bhat, S. K., Hiremath, S., \& Shenoy, N. (1994). Influence of handedness on the visual and auditory reaction time. Indian Journal of Physiology and Pharmacology, 38(4), 297299.

Schiff, S., Valenti, P., Andrea, P., Lot, M., Bisiacchi, P., Gatta, A., et al. (2008). The effect of aging on auditory components of event-related brain potentials. Clinical Neurophysiology, 119(8), 1795-1802.

Starr, A., Aguinaldo, T., Roe, M., \& Michalewski, H. J. (1997). Sequential changes of auditory processing during target detection: Motor responding versus mental counting. Electroencephalography and Clinical Neurophysiology, 105(3), 201-212.

Starr, A., Sandroni, P., \& Michalewski, H. J. (1995). Readiness to respond in a target detection task: Pre- and post-stimulus event-related potentials in normal subjects. Electroencephalography and Clinical Neurophysiology, 96(1), 76-92.

Verleger, R., Baur, N., Metzner, M. F., \& Śmigasiewicz, K. (2014). The hard oddball: Effects of difficult response selection on stimulus-related P3 and on response-related negative potentials. Psychophysiology, 51(11), 1089-1100. 